\title{
Comment favoriser la régulation biologique des insectes de l'échelle de la parcelle à celle du paysage agricole, pour aboutir à des stratégies de protection intégrée sur le colza d'hiver?
}

\author{
Muriel VALANTIN-MORISON \\ INRA, \\ UMR 0211 Agronomie, \\ F-78850 Thiverval-Grignon, \\ France \\ $<$ Muriel.Morison@grignon.inra.fr >
}

\begin{abstract}
How to enhance biocontrol of insects from the field scale up to agricultural landscape in order to propose integrated management strategies on winter oilseed rape?

Continuous increases in the use of synthetic fertilisers and pesticides in agro-ecosystems have led to an increase in crop production, but also to a simplification of landscape and a decline in biodiversity at the field, cropping system level. Changes at landscape level, such as regional farm specialisation, increases in field size and the removal of hedgerows and woodlots suppression, have strengthened this tendency towards decline. The loss of biodiversity in agro-ecosystems has increased the need for external inputs, as important functions are no longer provided by beneficial species. This has led to an apparently inevitable reliance on petrochemicals in production systems. However, many scientists are arguing that this reliance on pesticides could be considerably reduced by making better use of biotic interactions. This review explores, in the light of recent studies, possible ways to increase beneficial biotic interactions in agro-ecosystems, and to improve bio control pest management at field and landscape scales. This review also points out the possible integrated cropping system on oilseed rape and concludes on the future research that has to be engaged to achieve the goal of improvement the natural bio-control of pests. For example at field scale, it has been shown that the choice of cultivar, the sowing date and nitrogen fertilisation practices can be manipulated to prevent interactions between pests and crop, in either time or space. We have also highlighted that beneficial biotic interactions may result from appropriate changes to the habitats of beneficial species, mediated by soil management and cover or mixing species utilisation. Finally, changes achieved at landscape scale appear promising. However, these approaches frequently also present drawbacks that may not necessarily be outweighed by the beneficial effects. Endly, we propose the approaches to design integrated crop management systems to reduce the damage of pests. However, major research efforts are required to quantify and to use the effects of management practices and to improve our understanding of the interactions between the different levels.
\end{abstract}

Key words: natural bio-control of insect, integrated pest management, field scale, agricultural landscape, design and assessment of innovative crop management systems, low input cropping systems

recours à une forte mécanisation et à une utilisation massive d'intrants. Ces nouvelles pratiques agricoles ont bel et bien conduit à une augmentation de la productivité mais ont également entraîné des impacts environnementaux et sanitaires considérables. D'abord, I'utilisation massive de fertilisants et de pesticides est reconnue comme ayant des effets néfastes sur les écosystèmes, et sur la gestion durable des bio-agresseurs, via l'apparition de résistance (Aubertot tante des modes de conduite via le

Pour citer cet article : Valantin-Morison M. Comment favoriser la régulation biologique des insectes de l'échelle de la parcelle à celle du paysage agricole, pour aboutir à des stratégies de protection intégrée sur le colza d'hiver ? OCL 2012 ; 19(3) : 169-183. doi : 10.1684/ocl.2012.0455 
et al., 2005), et la santé humaine (Horrigan et al., 2002). Ensuite, l'agriculture moderne, à travers l'intensification des pratiques agricoles et la simplification des paysages, est reconnue comme responsable de nombreux impacts négatifs à différentes échelles : (i) aux échelles locales, avec l'augmentation des processus d'érosion, une moins bonne fertilité des sols ou une diminution de la biodiversité cultivée; (ii) aux échelles régionales, avec la pollution des eaux superficielles et souterraines ou l'eutrophisation d'écosystèmes aquatiques; (iii) et aux échelles globales, avec l'émission importante de gaz à effet de serre ou l'érosion de la biodiversité (Matson et al., 1997 ; Tilman et al., 2002 ; Stoate et al., 2001).

Pour toutes ces raisons, ce modèle $d^{\prime}$ agriculture est remis en cause (Tilman et al., 2002) et la production agricole mondiale doit faire face a un double défi : atteindre des objectifs de production importants pour nourrir la population mondiale tout en limitant les impacts sur l'environnement pour ne pas compromettre la disponibilité future des ressources. Le défi d'une agriculture plus durable, économiquement et environnementalement, nécessite de développer des systèmes de production écologiquement performants qui intègrent des connaissances du fonctionnement du champ cultivé, notamment celles sur les interactions milieu-composante biologique de l'agro-écosystème et celles sur les services rendus par la biodiversité depuis l'échelle de la parcelle à l'échelle du paysage.

Parmi les grandes cultures (céréales, oléagineux, pomme de terre et betteraves), le colza est une culture particulièrement exposée aux risques liés aux bio-agresseurs, ce qui se traduit par un indice de fréquence de traitement (IFT) de 6,1 en 2006. Bien que le poste herbicide constitue un coût important, $c^{\prime}$ est bien le poste insecticide, dont le coût est faible, qui occupe la part la plus importante dans le calcul de I'IFT. Alors que le colza n'occupe que $12 \%$ de la SAU grandes cultures, cette culture consomme $19 \%$ de I'IFT déployé sur la surface cultivée et $53 \%$ de cette part est consacré aux insecticides (EcoPhyto R\&D, 2009). Ceci s'explique par un cortège de ravageurs impressionnant (10 insectes coléoptères pour la plupart - Alford et al., 2003), des dégâts facilement reconnaissables pour une nuisibilité mal connue ou encore mal évaluée. L'intérêt agronomique de cette culture, son importance dans les successions du nord de la France et les objectifs ministériels de réduction des produits phytosanitaires, justifient très largement d'identifier, d'analyser des solutions " alternatives » et de construire et tester des itinéraires techniques intégrés, mobilisant plusieurs moyens de lutte. Pour cela, favoriser les processus de régulation biologique de ces ravageurs, apparaît comme une voie de recherche prometteuse et encore peu explorée. Tout I'enjeu consiste à analyser comment favoriser la régulation biologique de ces ravageurs. Analyser les interactions plantes bio-agresseur, connaître et comprendre les conditions qui favorisent la présence $d^{\prime}$ insectes auxiliaires ${ }^{1}$, sont des voies privilégiées. L'objectif de cet article est de mettre en lumière les résultats de travaux de recherche récents sur les moyens de défavoriser le bioagresseur et favoriser les ennemis naturels à l'échelle de la parcelle (partie 1) et favoriser les auxiliaires à l'échelle du paysage agricole (partie 2), afin de commencer à construire de nouveaux itinéraires techniques (partie 3).

\section{Comment favoriser les régulations biologiques à l'échelle de la parcelle}

\section{Concepts et mécanismes}

Comme le précise Prévost (2000), « en agronomie, les phénomènes de régulations biologiques concernent des flux liés aux cycles biologiques des systèmes vivants

\footnotetext{
${ }^{1}$ Les organismes auxiliaires en protection des cultures sont des antagonistes aux organismes nuisibles des cultures. Ils contribuent aux services écosystémiques rendus par la biodiversité. Dans le cadre de la protection des végétaux contre les ennemis des cultures, ces organismes auxiliaires sont aussi appelés organismes utiles ou bénéfiques. Ils peuvent être élevés et diffusés dans certaines cultures ou sylvicultures, ou ils peuvent être favorisés par certaines pratiques. Concernant les insectes du colza, les ouvrages de Alford et al., 2003 et de Williams, 2010a permettent d'obtenir les bases de connaissances biologiques sur l'ensemble des organismes auxiliaires, généralistes ou spécialistes et par les clefs d'identification de reconnaître un certain nombre d'entre eux.
}

produits en agriculture (flux d'éléments fertilisants, flux de matiere organique du sol, flux hydriques, flux de population $d$ 'adventices, flux d'ennemis des cultures, flux de pesticides, etc.) ". Dans le cas de la gestion des bio-agresseurs, I'objectif est donc d'arriver à optimiser le système en maximisant les capacités de régulations naturelles et de résilience de l'agroécosystème pour gérer durablement les populations de ravageurs. Par l'action de l'agriculteur, on cherche donc à anticiper sur les variations des flux d'organismes vivant nuisibles ou utiles, et à contrôler ces flux. C'est donc dans ce cadre conceptuel élargi de la régulation biologique, qui met en jeu les interactions couvert/bioagresseur/ennemis naturels/pratiques agricoles, que l'on illustrera les moyens de la favoriser.

On peut distinguer deux types d'approches, fondements du concept de protection intégrée qui sont (i) la combinaison des méthodes de lutte dans l'espace et dans le temps et (ii) la priorité donnée aux régulations naturelles pour gérer les populations de bioagresseurs et limiter leur nuisibilité. Gurr et al., 2003 parle alors d'approches ascendantes ou "bottom-up " qui consistent à utiliser les caractéristiques de la plante hôte pour limiter le développement des ravageurs et leurs dégâts, et les approches descendantes ou " top-down » qui consistent a stimuler les populations d'ennemis naturels. La première consiste à organiser un système de culture, qui évite le ravageur, qui est plus robuste face à une attaque, entraînant ainsi moins de pertes de production. La deuxième consiste à utiliser la biodiversité non cultivée afin de favoriser les ennemis naturels autour des parcelles et repose majoritairement sur I'utilisation de pratiques agricoles en local et dans un paysage, la gestion des habitats seminaturels et des aménagements paysagers autour des parcelles permettant de favoriser le développement et l'impact des auxiliaires (Gurr et al., 2003).

Les mécanismes mis en œuvre pour gérer les ravageurs et les maladies impliquent de travailler souvent à plusieurs échelles (plante, parcelle et paysage) et de gérer plusieurs interactions et des effets antagonistes. On résume souvent ces connaissances à l'aide d'un schéma de fonctionnement du champ cultivé (figure 1). Ce schéma montre de 
manière très générale les modes $\mathrm{d}^{\prime}$ action des différents leviers agronomiques à mobiliser. Par ce schéma, les processus explicités constituent des objectifs que l'on peut assigner aux systèmes de culture. Mais, chaque objectif peut être atteint par différents moyens. C'est l'ensemble combiné et logique de ces solutions agronomiques à effets partiels qui permettra d'atteindre l'objectif principal qui dans ce cas de figure est "favoriser la régulation biologique des insectes ravageurs". Cette formalisation est couramment employée, depuis plusieurs années, et se retrouve également dans des outils d'aide à la conception de systèmes de culture économes en intrants comme le guide Stephy (Attoumani-Ronceux et al, 2011).

Les interactions plantes insectes (l'élargissement à d'autres bio-agresseurs est tout à fait possible) peuvent se représenter en trois étapes :

i) avant l'attaque, on peut parler de potentiel initial d'infestation, c'est-à-dire les œufs ou cocons ou pupes d'insectes ravageurs présents sur la parcelle et laissés par la génération précédente. Ce potentiel d'organisme bioagresseurs peut être considéré pour les organismes inféodés à la parcelle, à un faible pouvoir de dispersion ou dont le cycle de vie n'exige pas de devoir quitter la parcelle pour passer au stade suivant ;

ii) lorsque l'infestation a lieu, cela recouvre tous les mécanismes qui permettent aux bioagresseurs d'entrer en contact avec la plante : il s'agit de la contamination par des spores de maladie, I'attaque des plantes par des insectes quels que soit leurs stades. Ces organismes peuvent venir de la parcelle ellemême ou des parcelles ou autres habitats alentours ;

iii) Cette étape recouvre la notion de dommages sur la culture à partir des dégâts réalisés par les bioagresseurs.

Les trois mécanismes qui interviennent lorsque l'on veut réduire les dommages des bio-agresseurs sont l'évitement, la modification de l'habitat, l'atténuation d'impact. Ils relèvent chacun de processus différents, complémentaires et à l'échelle de la parcelle mais aussi de la plante.

\section{Favoriser l'évitement}

Favoriser l'évitement consiste à mettre en place des combinaisons de pratiques qui permettent d'éviter que le bio-agresseur n'entre en contact avec la culture, par exemple, qu'un insecte n'attaque la plante lorsqu'elle y est sensible. Le décalage des dates de semis permet souvent de favoriser la désynchronisation des stades sensibles de la culture avec le cycle des bio-agresseurs.

Cet effet est connu de longue date sur les céréales et plantes oléagineuses : Dosdall et al. (1996) et Dosdall et Stevenson (2005) démontrent que les dégâts des petites altises de crucifères (Phyllotreta cruciferae) sont bien supérieurs sur des semis de printemps que sur des semis d'automne. Les dégâts occasionnés vont aller jusqu'à détruire le bourgeon terminal empêchant alors l'élongation de la hampe principale. Valantin-Morison et al. (2007) montrent également que des semis de colza d'été se traduisent par une proportion de plante contenant des larves de grosses altises (Psylliodes Chrysocephala) plus faible que des semis de Septembre. Mais des effets antago-

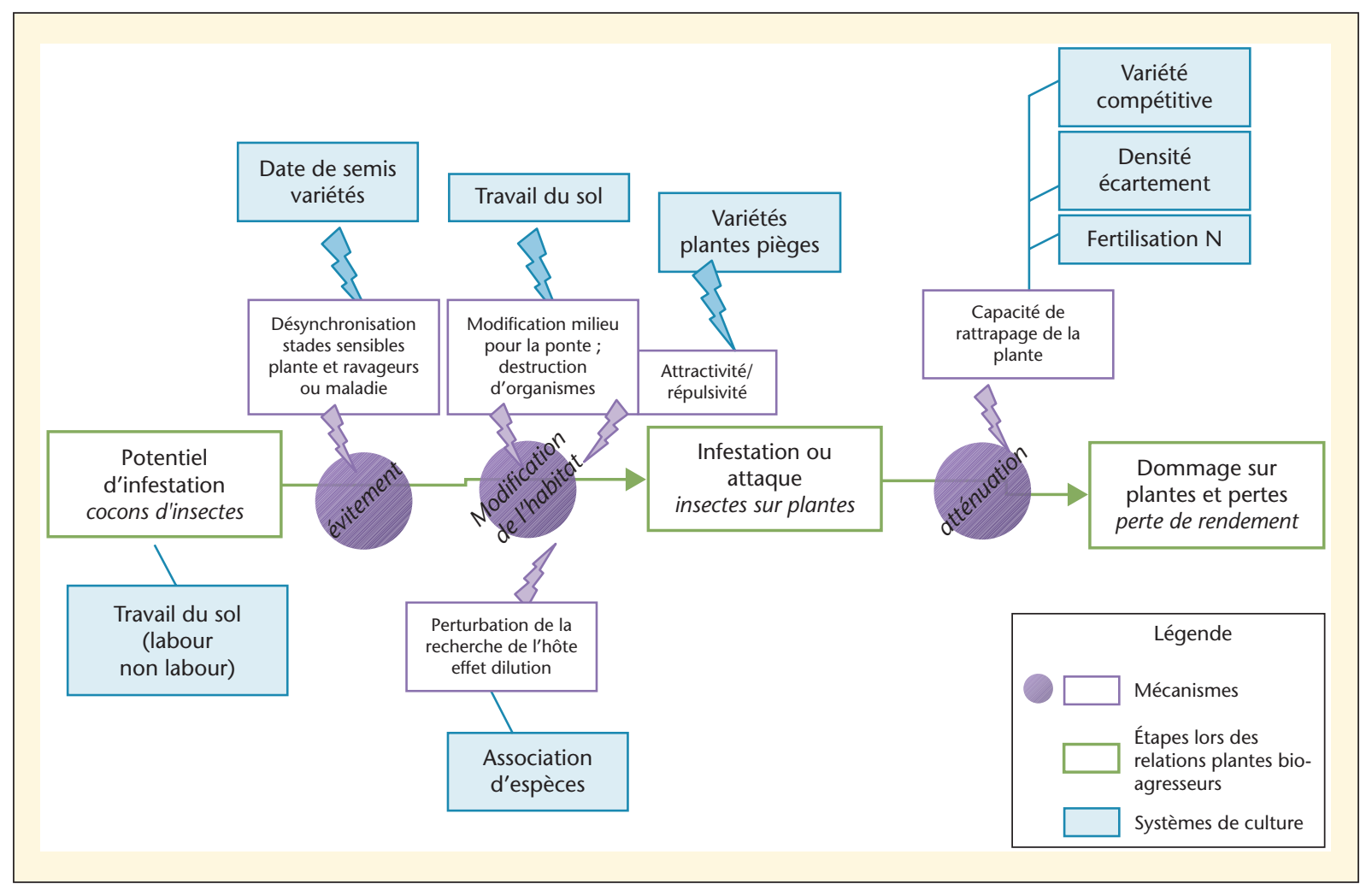

Figure 1. Schéma de fonctionnement des interactions couvert-bioagresseur et des effets attendus des leviers agronomiques à l'échelle parcelle. 
nistes peuvent apparaître, puisque cette même étude a montré que les dégâts de mouche du chou (Delia radicum) étaient alors plus importants sur des semis d'été. En outre, la date de récolte peut elle aussi avoir des conséquences importantes sur la faune auxiliaire du champ cultivé. Riechert et Lockley (1984) montrent que la récolte a un effet plus destructeur sur les araignées que les pesticides. Büchs (2003) parle également de coïncidence entre la récolte et l'activité maximale des prédateurs lors de la récolte des cultures d'hiver, ce qui n'est pas le cas pour les cultures de printemps. Bien que, parmi tous les leviers agronomiques, I'effet évitement via la date de semis soit l'un des plus important, il n'en reste pas moins vrai que (i) son effet est partiel (ii) il doit être raisonné en cohérence avec toutes les autres pratiques culturales.

Un autre levier pourrait permettre d'éviter l'attaque, de faire barrière à l'insecte : il s'agit de la résistance ou la tolérance variétale, mais on est encore loin d'y parvenir sur les crucifères. Kogan (1994) illustre que la résistance de la plante hôte à un insecte peut s'exprimer via des propriétés de la plante à restreindre la croissance de la population $d$ 'insectes ravageurs (par antibiose et/ou antixénose ${ }^{2}$ ) ou des capacités à compenser l'attaque. Sur colza, on observe encore assez peu d'exemples où l'on cherche à détecter, comprendre et utiliser cette "sensibilité ". Parmi ceux qui se sont orientés vers cette voie, on trouve : Ellis et Farrell (1995) et Dosdall et al. (1994) qui ont comparé la sensibilité variétale de plusieurs espèces et cultivars de colza et moutardes respectivement aux pucerons et à la mouche du chou et ont montré une variabilité plus forte entre espèces qu'entre culti-

\footnotetext{
${ }^{2}$ L'antibiose est une interaction biologique entre deux ou plusieurs organismes qui porte préjudice à au moins l'un d'entre eux ou bien une association antagoniste entre un organisme et les substances métaboliques produites par un autre. L'antixénose est un type $d$ 'interaction biologique entre une espèce de plante et un organisme prédateur. Dans cette relation, qualifiée aussi de "non-préférence ", la plante rejette le ravageur, par exemple un insecte, ou réduit les dommages qu'il pourrait lui causer, par différents caractères, morphologiques, physiologiques ou phénologiques, qui altèrent le comportement du ravageur et le portent à éviter la plante et à se tourner vers une autre espèce.
}

vars. Mais l'existence même d'une variabilité de réponse naturelle à l'infestation de la plante par des insectes ravageurs donne des espoirs pour la sélection variétale. Eickermann et al. (2011) ont réussi, par introgression de gènes provenant de la moutarde, à recréer des cultivars plus tolérants aux charançons de la tige du chou (Ceutorhynchus pallidactylus), ce qui démontre le potentiel de diversité génétique dans les espèces de crucifères pour la sélection de nouvelles variétés.

\section{Perturber le milieu/modifier I'habitat ou perturber le milieu}

Perturber le milieu consiste à défavoriser le microclimat nécessaire au développement d'une maladie, ou à défavoriser la germination, la levée d'une mauvaise herbe par le travail du sol ou l'allélopathie (Valantin-Morison et al., 2008, Ciag décembre 2008), ou à entraîner la mortalité des insectes enfouis dans le sol ou réduire la probabilité qu'un insecte retrouve son hôte en perturbant ses signaux olfactifs et visuels.

Le levier le plus connu est le travail du sol pour les insectes: il provoque des perturbations physiques majeures du milieu et peut avoir des effets directs et indirects sur la dynamique des populations, via son influence sur leur cycle de vie, et ainsi sur leurs dégâts (Altieri, 1999). Il enfouit des résidus de cultures contenant des larves ou des cocons d'insectes hibernant dans le sol de la parcelle, ce qui empêche alors aux organismes de terminer leurs cycles. L'absence de travail du sol peut aussi modifier le lit de semence et le rendre moins propice à la ponte de certains insectes.

Sur les neuf insectes les plus problématiques pour le colza, deux seulement terminent leur cycle dans la parcelle : le charançon de la tige du colza (Ceutorhynchus napi) et la Cécydomie (Dasineura brassicae). Pour ces deux espèces, bien qu'aucune étude ne le démontre, on peut supposer qu'un travail du sol profond réduit leur émergence l'année suivante. Pour les autres, l'influence du travail du sol n'est possible que via une modification de leur comportement de prospection de la plante hôte ou de ponte. En revanche, les populations $d^{\prime}$ arthropodes auxiliaires sont très sensible aux perturbations d'un travail du sol. La réduction de la densité d'arthropodes auxiliaires généralistes par des activités de travail du sol varie de 25 à $60 \%$ selon le taxon concerné (Thorbek et Bilde, 2004). Enfin, la période de labour, comme la date de récolte, évoquée ci-dessus, influe de manière importante sur la mortalité ces insectes auxiliaires (Büchs et al., 1999). Les prédateurs spécialistes, tels que les parasitoïdes de beaucoup d'insectes ravageurs du colza, sont très sensibles au travail du sol post-récolte, puisque la majorité d'entre eux hiverne dans les parcelles de colza après la récolte (Williams, 2010b ; Rusch et al., 2010). Nilsson (1985) et Hokkanen (1988) ont montré que la survie et donc l'émergence des parasitoïdes de méligèthes (Meligethes aeneus) du genre Tersilochus était réduite en cas de travail du sol profond. Enfin, Rusch et al. (2011a) ont réalisé une étude in situ sur 42 parcelles agricoles afin de déterminer à plusieurs échelles, les influences des pratiques agricoles et des habitats semi-naturels sur le parasitisme des larves de méligèthes. Ces travaux ont montré qu'à une large échelle (1 500 à 2000 m) le taux de parasitisme de deux Tersilochus (heterocerus et morionnelus) est positivement corrélé à la proportion en prairies et négativement corrélé à la proportion de colza labouré l'année précédente. On peut donc conclure que le travail du sol superficiel et le semis direct post-récolte du colza dans un rayon de $1500 \mathrm{~m}$ autour de la parcelle cible permet d'augmenter les années suivantes le taux de parasitisme des méligèthes par deux espèces de parasitoïdes majeurs.

La réduction du travail du sol a d'autres conséquences car la teneur en matière organique, la localisation d'un mulch et des résidus de cultures, la structure du sol, sa porosité, sont entièrement modifiés, ce qui a des conséquences sur la recherche de sites de ponte d'insectes ravageurs, mais ce qui encourage également la colonisation de la parcelle par de nombreux insectes auxiliaires (carabes, staphylins et araignées) en créant un milieu moins perturbé (Holland, 2004). De nombreuses études et revues sur diverses cultures, dont le colza, évoquent cet effet positif du non travail du sol sur les populations d'insectes auxiliaires généralistes (Kromp, 1999 ; Landis et al., 2000 ; Schmidt et al., 2004 ; Pullaro et al., 2006). Quelques-unes de ces mêmes études vont jusqu'à montrer une décroissance des insectes ravageurs: Zehnder et 
Hough-Goldstein (1989), Schmidt et al. (2004), Pullaro et al. (2006). D'autres travaux montrent également que le mulch de surface pouvait causer des perturbations dans la phase de recherche et d'approche des insectes ravageurs tels que les petites altises (Phyllotera sp) et mouche du chou (Delia radicum): Dosdall et al. (1999), Mabbett (1991), Finch et Collier (2000).

Néanmoins l'effort de démonstration de l'effet positif du non travail du sol sur les insectes ravageurs via une augmentation du nombre et de la diversité des insectes auxiliaires généralistes ou spécifiques est encore à faire et les premiers résultats encourageants initient de récents travaux de recherche. En outre, il ne faut pas négliger l'impact de l'absence du travail du sol sur d'autres organismes comme les mauvaises herbes, souvent favorisées (Büchs et Katzur, 2004), et les limaces, également plus nombreuses (sur l'orge, voir Mabbett (1991)). Par conséquent, aucun consensus n'est encore admis sur les bénéfices du non-travail du sol et la multiplicité des outils et l'innovation en matière de mécanisation nous invitent à être précis dans la description des types de travaux du sol. En outre, les effets antagonistes d'une seule et même pratique pointe bien le fait que la traduction de ces connaissances en stratégies de protection intégrée ne peut se satisfaire d'une juxtaposition des solutions alternatives. Combiner le choix de la date de semis, du meilleur travail du sol ou du semis direct, du choix variétal, etc., passe par une phase de conception-évaluation où les solutions sont assemblées de manière cohérente au regard d'objectifs complémentaires.

Le choix variétal ou le choix d'espèces est également un autre moyen de perturber le milieu, car il peut influencer la recherche de la plante hôte. Les différents comportements des insectes, ravageurs ou auxiliaires des cultures, comme la recherche de I'hôte, de nourriture, l'oviposition sont affectés par des signaux chimiques (Free et Williams, 1978) ou visuels, comme la couleur, la taille, l'architecture de la pante, la structure de la feuille (Hopkins et Ekbom, 1996, 1999 ; Ulmer et Dosdall, 2006). De nombreux exemples sont développés sur les crucifères en raison de la présence de nombreux signaux chimiques de la famille de métabolites secondaires, les glucosinolates: les alkenyl glucosinoltaes, qui se dégradent en isothiocyanates volatiles sont plus attractifs pour la sélection de la plante hôte.

Sur colza, ce sont les travaux d'entomologistes de Rothamsted (Cook et al., 2002, 2006 ; Barari et al., 2005) qui se préoccupent les premiers des effets de la navette (Brassicae rapa) sur la présence et les dégâts de nombreux insectes ravageurs mais aussi auxiliaires. De nombreux travaux existent maintenant et concluent pour la plupart que la navette est plus attractive que le colza, que ce sont les différences de teneur en glucosinolates et de précocité à floraison qui expliquent ces différences de comportement. Ces différences peuvent être exploitées dans une stratégie push pull (Cook et al., 2007), qui consiste à attirer les insectes dans une zone particulière de la parcelle, souvent la bordure, et à les empêcher d'atteindre la culture de rente. Le recours à des bordures de navette ou de variétés plus précoces a été testé il y a déjà plusieurs années en parcelles agricoles, avec des résultats encourageants (Valantin-Morison et Quéré, 2006 ; Rusch et Valantin-Morison, 2008; Valantin-Morison et Meynard, 2012). Les simulations d'arrangements paysagers, basées sur un modèle spatialement explicit a permis de montrer que l'agencement de bordures de plantes pièges étaient l'arrangement optimal pour assurer la capture des insectes ravageurs lors de la floraison (Potting et al., 2005). La prise en compte de ces effets de bordures de plantes piège doit alors dépasser l'échelle de la parcelle cultivée, ce qui a été réalisé dans le cadre de la construction d'un prototype de modèle spatialement explicit (voir section suivante).

Des plantes de couverture, plantes compagnes, ou associations d'espèces permettent d'augmenter la biodiversité dans le champ cultivé, ce qui permettrait d'augmenter les ressources pour les insectes auxiliaires généralistes et de perturber le milieu pour les insectes ravageurs. L'ensemble de ces espèces végétales est souvent regroupé sous le terme de plantes de services et on peut distinguer les plantes de couverture qui sont détruites pendant ou après la récolte de la plante de rente et celles, qui constituent un couvert vivant permanent. On trouve communément dans la littérature, la distinction de trois mécanismes qui tentent d'expliquer les effets liés à l'introduction d'une autre espèce dans un couvert de plante cultivée : i) un effet de la qualité de l'hôte, car l'architecture, le microclimat sont différents du couvert pur, ce qui peut provoquer un changement de comportement des insectes ravageurs (et auxiliaires), se traduisant par un effet "leurre ", une attractivité modifiée ;

ii) un effet " concentration des ressources", très lié au premier (Tahvanainen et Root, 1972 ; Root, 1973), qui émet I'hypothèse que la probabilité pour un insecte ravageur de trouver la plante hôte dans un couvert monospécifique est plus grande que dans un couvert plurispécifique ;

iii) un effet " ennemis naturels" (Root, 1973), qui émet l'hypothèse que la réduction du nombre de ravageurs peut être indirectement liée à la présence plus importante d'ennemis naturels, attirés par davantage de ressources.

En grandes cultures (particulièrement céréales/légumineuses) ou en cultures pérennes (arboriculture, viticulture), ces innovations ont fait l'objet de très nombreuses études, impossibles à reprendre fidèlement ici (par exemple : Landis et al. (2000); Ferron et Deguine (2005), Wyss (1995), Pickett et Bugg (1998), Altieri et Nicholls (2004), Broad et al. (2008)). Beaucoup ont montré une réduction significative des insectes nuisibles dans les systèmes de cultures associées, comparativement aux monocultures des mêmes espèces. L'ensemble de ces travaux a été résumé dans la synthèse bibliographique de Andow (1991) et Malezieux et al. (2008). Ainsi, Andow (1991) a analysé 209 études sur les associations, impliquant 287 espèces d'insectes parasites différentes, et en a conclu que les insectes étaient significativement moins importants dans $52 \%$ des cas (149 espèces) comparé aux monocultures, et qu'ils étaient considérés comme plus importants dans $15 \%$ des cas (44 espèces). En effet, il ne faut pas négliger que l'effet réducteur n'est pas systématique et l'on peut parfois observer une aggravation de l'attaque (Pfiffner et Wyss, 2004).

Mais concernant le colza, très peu d'études existent aujourd'hui et sont encore peu démonstratives sur les réels avantages du mélange d'espèces. Weiss (1994) ont observé un effet d'un mélange pois/colza de printemps (les auteurs canadiens parlent de Canola) sur les attaques de petites altises (Phyllotreta cruciferae). Mais, ni Butts et al. (2003) ni Hokkanen (2008) n'ont observé d'effet 
significatif de mélanges d'espèces contenant du colza sur respectivement les prédateurs généralistes rampant ou les attaques de méligèthes ou par le parsitisme de leurs larves. Hummel et al. (2010) montrent même une réduction du taux de parasitisme en cas d'association colza-blé. Enfin, alors que les associations céréales-légumineuses sont souvent étudiées, les associations crucifères légumineuses sont quasiment absentes de la littérature. Les quelques travaux comprenant des crucifères en association avec des céréales se résument à I'analyse du cycle de l'azote et de la croissance (Andersen et al., 2007) ou à I'analyse de I'influence sur un seul type d'insecte (Finch et al., 2003 ; Björkman et Eklund, 2006). L'engouement pour les influences supposés positifs de la biodiversité sur la régulation biologique a conduit à initier de très récents travaux de recherche et de développement dont l'objectif est d'analyser les avantages et les inconvénients de mélanges d'espèces colza-légumineuses (Valantin-Morison et al., 2011 ; Cetiom, 2011).

\section{Atténuer l'impact du bioagresseur}

L'atténuation de l'impact consiste à réduire I'amplitude des dégâts lorsqu'une attaque intervient en augmentant la capacité de rattrapage des plantes. Le plus souvent, il s'agit de jouer sur la vigueur du couvert en jouant beaucoup sur la fertilisation azotée. On peut par exemple favoriser la compétition du couvert pour réduire l'enherbement, favoriser la compensation de la plante pour réduire l'intensité des pertes de production, modifier l'architecture de la plante, ce qui induit des modifications dans les interactions avec les insectes.

La gestion de la disponibilité en azote et le choix variétal sont les leviers majeurs pour atténuer l'impact des bioagresseurs sur les dommages. Des études anciennes et relativement nombreuses montrent que le colza, comme beaucoup $d$ 'autres crucifères sont capables de compenser les dégâts occasionnés par des insectes herbivores ravageurs via la production de ramifications secondaires, tertiaires, quaternaires, qui produisent de nouvelles fleurs et de nouvelles siliques, via l'augmentation du nombre de grains par siliques, via l'augmentation du poids de mille grains (Williams et Free, 1979 ; Lerin, 1988).
L'insecte le plus souvent étudié pour démontrer cette capacité de compensation est le méligèthe (Meligethes aeneus). II se nourrit du pollen des fleurs et détruit les boutons floraux lorsque les fleurs ne sont pas écloses, entraînant la chute de la fleur. Ces dégâts entraînent la production de nouvelles ramifications secondaires (Nilsson, 1994; Lerin, 1988) et davantage de siliques par ramification (Podlaska et al., 1996). Un travail récent (Jullien et al., 2011) à l'échelle de la plante entière a cherché à comprendre comment les dynamiques sources-puits de la plante se mettaient en place et explicitaient la compensation d'une réduction de boutons floraux, et ce dans différentes conditions de fertilisation azotée avec différents cultivars. Les attaques de méligèthes ont été simulées par différents types d'ablation de fleurs (ablation drastique sur la hampe florale, plus dispersée dans le couvert). Les résultats de cette étude montrent que, suite à une ablation de boutons floraux, la plante met en place un nombre de boutons floraux deux à trois fois supérieur au nombre de boutons floraux supprimés. Après une phase d'avortement d'une partie de ces boutons floraux supplémentaires, le nombre de boutons floraux restant permet d'atteindre un nombre de siliques équivalent à celui des plantes témoin mais avec un retard. La compensation de la perte de boutons floraux se traduit donc par un retard de la demande reproductrice. Cette modification de la morphogenèse modifie les composantes du rendement de manière très variable en fonction des génotypes et des fertilisations azotées. Si toutes les composantes du rendement sont modifiées, deux sont principalement concernées : le nombre de ramifications secondaires dont la contribution au rendement augmente de manière significative, et le nombre de siliques par ramification. Au final, les rendements sont équivalents entre plantes ayant subi différents traitements d'ablation et plantes témoin, voire, en tendance, supérieurs en situation de forte fertilisation azotée. On confirme ici et on démontre à l'échelle de la plante entière que l'intensité de la fertilisation azotée pouvait induire des phénomènes de compensation tels que mêmes de fortes attaques de méligèthes, simulées par une ablation de la hampe florale, ne se traduisaient pas par une réduction de rendement. Cela traduit bien cette notion d'atténuation d'impact, que d'autres études à l'échelle du couvert avaient remarqué (Podlaska et al., 1996 ; Valantin-Morison et al., 2007). Nilsson (1994) a en outre démontré que les méligèthes sélectionnaient les boutons floraux les plus larges, ce qui peut potentiellement être influencé à la fois via la variété et la fertilisation azotée. Enfin, d'autres insectes sélectionnent les plantes en fonction de leur hauteur, diamètre. $C^{\prime}$ est le cas respectivement du charançon de la tige du colza (Ceuthorhynchus napi) et de la mouche du chou (Delia radicum) (Dosdall et al., 1996 ; Valantin-Morison et al., 2007). Ainsi même si la fréquence d'attaque de la mouche du chou était supérieure dans les cas de semis avancé, les plantes étaient souvent plus vigoureuses, présentaient un diamètre de pivot plus élevé et se révélaient donc moins sensibles à la destruction du pivot en cas d'attaque par la mouche du chou (Valantin-Morison et al., 2007).

Ces éléments de connaissance font référence à la notion de nuisibilité des attaques et l'on perçoit à travers ces résultats qu'une fréquence ou une intensité d'attaque d'un insecte n'aura pas les mêmes conséquences sur le rendement selon l'état du couvert de la plante, lui-même influencé par les pratiques culturales. Cette interaction couvert/bioagresseur/pratiques culturales est fondamentale dans la protection intégrée. Pourtant aujourd'hui encore, les seuils de nuisibilité sont exclusivement basés sur un nombre d'insectes (Williams, 2010b) et sont pour les méligèthes éminemment variables d'un pays à l'autre, voire inexistant pour bon nombre d'entre eux, puisque c'est dès l'apparition d'un seul individu que le déclenchement du traitement est conseillé pour les charançons du bourgeon terminal, de la tige du colza, des siliques (Pilorgé et al., 1997). Une meilleure connaissance des conditions de croissance des plantes au moment de l'attaque et des conséquences sur les pertes de rendement permettraient sans aucun doute de revoir ces seuils de nuisibilité et de les complexifier en tenant compte d'indicateurs du couvert.

\section{Conclusion}

L'ensemble de ces travaux montrent combien les interactions couvert/ bioagresseurs et ennemis naturels constituent la clef d'une agriculture plus 
durable et tout l'enjeu consiste à piloter ces interactions via les pratiques agricoles (Médiène et al., 2011). Mais on comprend bien que chaque levier a un effet très partiel et qu'ils doivent être associés de manière cohérente pour bénéficier $d$ 'une synergie et assurer une production agricole rentable. On suspecte aussi assez intuitivement que l'échelle de la parcelle ne suffit pas à aborder la régulation biologique d'organismes mobiles comme les insectes ravageurs et les auxiliaires des cultures : deux coléoptères sur les neuf plus problématiques restent inféodés à la parcelle, les autres ayant besoin d'autres types d'habitats pour assurer leur survie et neuf organismes auxiliaires sur dix ont besoin des habitats semi-naturels pour survivre.

\section{Comment favoriser les régulations biologiques à l'échelle du paysage agricole}

Comme dit en introduction, I'agriculture intensive a conduit dans beaucoup de régions européennes à une simplification, du paysage agricole et une réduction des zones non cultivées, d'où une fragmentation des paysages ruraux. Plusieurs travaux montrent que les interactions biotiques entre insectes utiles ou ravageurs doivent être regardées à une échelle spatiale plus grande que la parcelle (Dunning et al., 1992 ; Fahrig et Merriam, 1994 ; Bianchi et al., 2006).

\section{Concepts et mécanismes}

Favoriser les régulations biologiques à l'échelle du paysage recouvre principalement les moyens de lutte biologique, qui constitue une méthode importante pour la protection intégrée (Stern et al., 1959). II constitue le deuxième pilier (approche top-down) de la protection des cultures. II existe un nombre important de définition de la lutte biologique, nous retiendrons ici celle d'Eilenberg et al. (2001): "The use of living organisms to suppress the population density or impact of a specific pest organism, making it less abundant or less damaging than it would otherwise be ${ }^{\prime \prime}$. La lutte biologique recouvre quatre grandes stratégies qui utilisent des modes d'action bien différents (voir Rusch (2010) pour la synthèse). Nous focaliserons les exemples sur la lutte biolo- gique par conservation et gestion des habitats (Conservation biological control). Elle vise à préserver et augmenter les populations d'auxiliaires naturellement présents dans les agroécosystèmes grâce à la conservation et la gestion adaptée des éléments du paysage (Landis et al., 2000). Dans cette approche, on cherche à maximiser l'impact des ennemis naturels en adoptant des pratiques agricoles et des gestions d'habitats semi-naturels qui favorisent le développement des populations d'auxiliaires en leur fournissant des ressources écologiques fondamentales (Landis et al., 2000 ; Gurr et al., 2003). C'est de celle-ci qu'il est question dans l'illustration de la figure 2.

Dans cette approche, les mécanismes sont centrés sur les liens ravageursennemis naturels-champ cultivé et efficacité de la régulation biologique. Il faut alors s'interroger sur le rôle du paysage agricole au sens large, c'està-dire incluant à la fois les parcelles cultivées, assorties de leurs pratiques et les habitats semi-naturels non cultivés. Les trois rôles clés que joue le paysage sur les populations d'ennemis naturels et les insectes ravageurs sont: (i) faciliter le déplacement des organismes auxiliaires pour échapper aux zones perturbées que sont les parcelles agricoles, (ii) la création ou l'entretien d'habitats refuge, (iii) l'apport des sources de nourritures soit sous forme de pollen ou de nectar, soit sous forme de proies ou d'hôtes alternatifs (Landis et al., 2000 ; Bianchi et al., 2006).

En outre de manière générale, l'organisation des parcelles agricoles et autres habitats dans un paysage peut influencer la rencontre ravageurs/ennemis naturels et favoriser ou non la colonisation rapide des auxiliaires des cultures dans le milieu cultivé. Dunning et al. (1992) ont formalisé quatre grands types de processus affectant la dynamique des populations à l'échelle d'un paysage: la complémentation, la supplémentation, les relations sourcepuits et l'effet voisinage. La complémentation apparaît lorsqu'une espèce requiert au moins deux ressources différentes (e.g. pour l'alimentation, la reproduction ou I'hivernation) situées dans des types d'habitats différents au cours de son cycle de vie. L'espèce en question devra donc se déplacer entre ces deux types d'habitats pour assurer sa survie. Dans le cas où les habitats contiennent des ressources substituables (même ressource ou même fonction) mais dans des quantités limitées, alors l'organisme devra se déplacer entre les différentes taches d'habitats: c'est la supplémentation. Les relations sourcepuits correspondent aux mécanismes décrits précédemment durant lesquels la survie d'une population au sein de certaines tâches puits dépendent de l'immigration d'individus provenant de taches sources. Enfin, les relations de voisinage impliquent que l'abondance d'une espèce d'une tache d'habitat donnée est plus affectée par les caractéristiques des taches contiguës que par celles des habitats plus distants.

Modifier la structure du paysage pour favoriser la colonisation des parcelles par les insectes auxiliaires

Ces processus de colonisation sont capitaux pour comprendre les dynamiques des populations et à la fois la taille des habitats et de la distance entre les habitats influent la distribution des espèces. On parle alors souvent de connectivité des éléments du paysage entre eux, ce qui permet dans des paysages fragmentés d'assurer des lieux de connexion entre différents patch d'habitats favorables aux insectes auxiliaires. Le degré de connectivité d'un paysage pour une espèce donnée dépend de deux facteurs : (i) la configuration spatiale et de l'abondance des habitats dans le paysage, et (ii) I'aptitude à se disperser de l'espèce étudiée. On admet communément que les habitats semi-naturels $^{3}$ (forêts, bordures de champ, bandes enherbées, jachères, fleuries ou non, linéaires de haies) sont

\footnotetext{
${ }^{3}$ Habitat naturel : En Europe, la directive Habitats Faune Flore (DHFF), définit la notion $d^{\prime}$ 'habitat naturel par " un espace homogène par ses conditions écologiques (compartiment stables avec ses conditions climatiques, son sol et matériau parental et leurs propriétés physico-chimiques), par sa végétation (herbacée, arbustive et arborescente), hébergeant une certaine faune, avec des espèces ayant tout ou partie de leurs diverses activités vitales sur cet espace " En d'autres termes, un habitat naturel (ou semi-naturel) est " un ensemble reconnaissable, formé par des conditions stables (climat, sol, relief) et par une biocénose caractéristique aussi bien végétale qu'animale »
} 


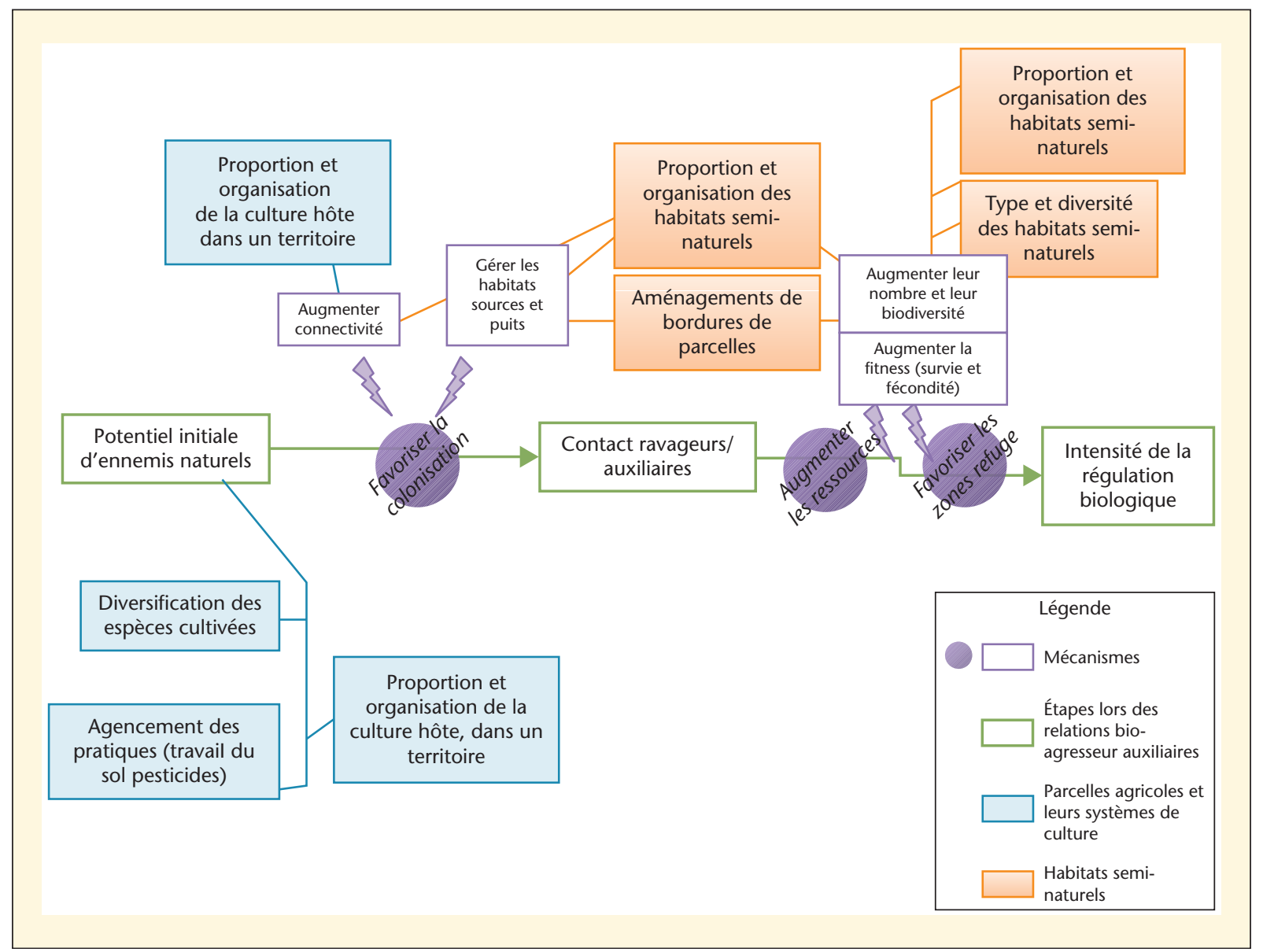

Figure 2. Schéma de fonctionnement des interactions insectes-ennemis naturels-parcelles cultivées et des effets attendus des habitats semisnaturels et des pratiques agricoles à l'échelle du paysage.

les zones sources $d^{\prime}$ insectes auxiliaires mais aussi d'un certain nombre d'insectes ravageurs (Denys et Tscharntke, 2002 ; Marshall, 2004). Or, il a aussi souvent été observé que les distances de dispersion des insectes bénéfiques spécifiques ou généralistes sont bien plus faibles que les insectes ravageurs (Roland et Taylor, 1997 ; Zabel et Tscharntke, 1998). On comprend alors aisément comment la raréfaction et l'éloignement (donc la fragmentation) des habitats semi-naturels peuvent réduire la connectivité des habitats favorables aux insectes auxiliaires et défavoriser la colonisation des parcelles par ces mêmes organismes. Mais les choses ne sont pas toujours si simples et il ne faut pas oublier que la simplification des paysages agricoles n'était toujours guidée par l'emploi facilité et systématique de machines agricoles, mais aussi par la présence de zones refuges pour certains bio-agresseurs des cultures... D'ailleurs, une revue de Bianchi et al. (2006) montre que la pression des insectes ravageurs toutes cultures confondues était réduite dans $45 \%$ des situations où le paysage était qualifié de complexe, alors que dans $75 \%$ de ces situations, où le paysage était complexe, le nombre et l'activité des ennemis naturels étaient augmentés.

Concernant les insectes du colza, les insectes auxiliaires les plus couramment étudiés sont les insectes spécifiques, tels que les parasitoïdes. Thies et al. (2003) et Thies et Tscharntke (1999) ont été les premiers à montrer que les paysages complexes permettaient un taux de parasitisme plus élevé sur les larves de méligèthes, ce qui pouvait potentiellement expliquer la réduction des dégâts de cet insecte. Un travail récent en parcelle agricole et évoqué préalablement a été plus loin dans l'analyse des déterminants du parasitisme des larves de méligèthes (Rusch et al., 2011a ;
Rusch, 2011b). Sur les 42 parcelles agricoles, de Normandie, il a été montré que le taux de parasitisme des larves de méligèthes oscillait entre 0 et $98 \%$ et était dû à trois ichneumonidés : Tersilochus heterocerus, Tersilochus morionellus, Phradis interstitialis. Le taux de parasitisme était fortement et positivement corrélé à la proportion en prairies et en forêt et à la proximité au colza de l'année précédente, dans un rayon de $250 \mathrm{~m}$ autour des parcelles cibles. En revanche à une plus large échelle (1 500 à 2000 m) le taux de parasitisme de Tersilochus heterocerus est positivement corrélé à la proportion en prairies et négativement corrélé à la proportion de colza labouré l'année précédente. L'hypothèse le plus souvent privilégiée pour expliquer l'effet positif des prairies et forêt est la disponibilité en ressource florale plus importante dans des paysages complexes, ce qui favoriserait la survie des ennemis naturels. 
Mais là encore les paysages plus complexes, dont la connectivité pour les organismes auxiliaires est potentiellement plus grande sont parfois aussi plus propices aux insectes ravageurs. Les liens entre la complexité des paysages agricoles et l'intensité des dégâts de charançons, de cécydomies et de méligèthes ont été étudiés par Thies et al. (2003), Zaller et al. (2008a, b) et Rusch et al. (2011a, b). Dans ces études les résultats sont contrastés : alors que Thies et al. (2003) observent que les dégâts de méligèthes sont plus importants dans les paysages simplifiés que dans les paysages complexes, Zaller et al. (2008a, b) et Rusch et al. (2011b) montrent le contraire. Ce dernier sur les 42 parcelles agricoles de colza la densité de méligèthes $/ \mathrm{m}^{2}$ oscillait entre 11 et 628 individus $/ \mathrm{m}^{2}$, avec un niveau de dégâts compris entre 3 et $58 \%$ de boutons détruits. Nous avons montré que la densité de population de méligèthes, ainsi que leurs dégâts étaient essentiellement déterminés par la complexité du paysage en particulier par la proportion de forêt et en prairies ainsi que l'état azote de la culture. Plus particulièrement, les analyses statistiques montrent une corrélation positive entre la proportion en forêt, en prairies et l'abondance et les dégâts du ravageur. Ces auteurs suggèrent que les paysages complexes contiennent davantage d'habitats "sources ", propices à la survie de ce ravageur. Rusch et al. (2011c) montrent que c'est effectivement le cas. Ulmer et Dosdall (2006) montrent aussi que les charançons des siliques ont besoin d'habitats boisés pour réaliser leur diapause hivernale. Ces deux exemples illustrent bien les concepts de complémentation, évoqués plus haut et la notion de sources/puits.

Les différences de résultats, obtenus sur méligèthes entre différents auteurs pointent les différences d'approches méthodologiques et aussi les difficultés expérimentales qui parfois réduisent la généricité des résultats. En outre, très peu d'autres études existent sur d'autres insectes, comme les altises ou les charançons de la tige. Or, ces deux insectes ont des comportements très différents : les premières font une diapause estivale avant l'attaque hors des parcelles cultivées alors que le deuxième reste à l'état de cocon après la récolte jusque l'attaque de l'année suivante. On peut donc supposer que la structure du paysage, la densité d'habitats non cultivés et le voisinage des parcelles auront des effets très différents sur leurs attaques. Les études qui analysent les effets du paysage et des pratiques ou qui reviennent sur des bases biologiques du cycle de développement de ces insectes et de leurs ennemis naturels sont inexistantes de la littérature.

\section{Augmenter les ressources et les zones refuges}

Les habitats non cultivés sont reconnus comme source de pollen ou de nectar, essentiels à de nombreux insectes (dont le groupe des ichneumonidés, guêpes parasitoïdes auquel appartient de nombreux insectes auxiliaires des ravageurs du colza) (Sotherton, 1984 ; Pickett et al., 2000 ; Denys et Tscharntke, 2002). Les zones boisées, ou linéaires d'arbres, protègent des insectes bénéfiques à plusieurs titres : un microclimat plus tempéré (Rahim et al, 1991), de faibles perturbations anthropiques; ils constituent souvent des zones d'hivernation ou d'estivation propice pour tous ces organismes ravageurs ou utiles (Rusch et al., 2010). Bien que la démonstration soit délicate, des études, via des analyses statistiques spatialisées montrent combien l'importance quantitative locale et à plus large distance influencent le nombre et la survie des organismes utiles (Rusch et al, 2011a, b ; Plantegenest et al., 2007).

Ainsi le recours à des zones tampons, haies arbustives, bandes fleuries, bordures aménagées dans un paysage constitue des moyens pour améliorer le niveau et la proximité des ressources et des refuges. Mais, la composition floristique est un élément capital pour assurer une meilleure attractivité des insectes utiles (Rebek et al., 2006). C'est pourquoi, de nombreuses études se sont orientées vers la détermination et le test de mélanges floristiques pour héberger et fidéliser davantage d'insectes auxiliaires, tout particulièrement les prédateurs spécialistes et parasitoïdes, souvent gros consommateurs de nectar et de pollen (Wackers, 2004 ; Pontin et al., 2006). Les bandes fleuries les plus diversifiées hébergent souvent le plus de diversité d'arthropodes (Lagerlof et Wallin, 1993). Enfin, la littérature pointe aussi l'importance de l'âge de la bordure pour assurer une bonne efficacité de régulation biologique (Frank, 1996;
Büchi, 2002). Büchi (2002) a justement montré sur colza que le parasitisme par Tersilochus heterocerus était significativement plus élevé lorsque les parcelles jouxtaient une ancienne bordure de fleurs sauvages plutôt qu'une prairie. Thies et Tscharntke (1999) ont testé des bordures de mêmes compositions mais d'âges différents et ont montré que la mortalité des méligèthes via le parasitisme était plus importante lorsque les bordures avaient au moins six ans d'âge.

\section{Conclusion}

Aujourd'hui, aucune "pratique » de gestion des bordures, aucun consensus sur des " aménagements paysagers " ne fait l'unanimité pour favoriser l'efficacité de la régulation biologique par les insectes auxiliaires tout en limitant la prolifération des insectes ravageurs et tout en assurant une production agricole rentable. Dans la communauté scientifique, I'influence de la complexité du paysage, le recours à des aménagements de bordures, tels que les bandes fleuries, pièges, enherbées, les surfaces compensatrices font encore débat quant à l'intérêt pour assurer l'efficacité de la régulation biologique des insectes. Certes beaucoup d'études montrent une augmentation du nombre et de la diversité des ennemis naturels mais peu encore vont jusqu'à démontrer une réduction du nombre d'insectes ravageurs et encore moins jusqu'à la réduction des dégâts et des dommages. Pourtant, c'est maintenant indispensable. En effet, si l'on ambitionne de produire durablement et de co-construire avec la participation de la filière agricole des stratégies de protection intégrée, l'efficacité de la régulation biologique des insectes, via l'augmentation d'insectes utiles à ces échelles supraparcellaire, doit être quantifiée. En outre, on peut aussi largement regretter que dans la majorité des études citées, la prise ne compte des pratiques culturales à de l'ensemble des parcelles agricoles d'un paysage soit négligée. Néanmoins, ce front de science avance et l'orientation de la recherche vers I'agro-écologie permettra probablement de répondre demain à ces questions.

En outre, ces résultats et ces questions de recherche posent de nouvelles questions méthodologiques : comment démontrer à de telles échelles I'influence de tels habitats? Le recours à «l'expérimentation » est quasiment 
impossible et I'on doit par méthodes statistiques appropriées exploiter la diversité (Vinatier et al., 2011) des paysages agricoles pour analyser les influences des hétérogénéités spatiales.

\section{Quelles stratégies de protection intégrée pour favoriser la régulation biologique des insectes}

\section{Concepts de l'agronomie systémique à mobiliser}

L'ensemble des "solutions » individuelles évoquées ci-dessus ne sont pas tous utilisables, bien que nous ayons mis I'accent sur ceux qui seront mobilisables facilement; en outre il ne suffit pas de les cumuler pour obtenir des itinéraires techniques ou des systèmes de culture intégrés. Enfin, favoriser la régulation biologique des insectes ravageurs $n$ 'est par le seul objectif que doit remplir les systèmes de culture de demain. II faut donc bien adopter une démarche de conception plus générique, qui se préoccupe de l'ensemble des bioagresseurs, pour aboutir à des stratégies de protection intégrée.

La conception de solutions nouvelles peut se réaliser de trois grandes manières, souvent complémentaires : (i) soit par prototypage (Lançon et al., 2007) c'est-à-dire en mobilisant plusieurs acteurs et à partir de leur connaissance experte, concevoir des systèmes prototypes qui sont à leur tour évalués, (ii) soit " pas à pas " par des aller-retour entre conception et évaluation au champ en mobilisant peu d'experts mais les connaissances agronomiques acquises par ailleurs et souvent via un schéma de fonctionnement, (iii) soit avec l'aide de modèles simples (bilan $\mathrm{d}^{\prime}$ azote, bilan hydrique, etc.) ou complexes (modèle de cultures; David et al., 2005 ; Barbottin et al., 2006) ou des modèles spécifiquement construits pour classer et identifier les systèmes de culture les mieux adaptés (Loyce et al., 2002).

\section{Concevoir, expérimenter, évaluer}

Afin de concevoir et évaluer des itinéraires techniques intégrés, nous avons dans les années précédentes mobilisé une méthode de conception " pas à pas ", qui recours à une boucle dite de progrès " conception/évaluation des systèmes de culture " (Meynard et al., 2001) : diagnostic agronomique, construction d'un schéma conceptuel, conception et évaluation a priori et in situ (Valantin-Morison et Meynard, 2012). Tout au long de cette boucle, les connaissances nécessaires à l'interprétation des résultats ou à la mise en œuvre des itinéraires techniques sont mobilisées et sont de plusieurs natures : connaissance experte issue d'un collectif, connaissances scientifiques sur les interactions couvert-milieu ou couvert-bioagresseur, évoquées dans les premières parties de ce texte. Pour chaque itinéraire technique, on identifie des objectifs intermédiaires pour caractériser le couvert ou les relations plantes-bioagresseurs: par exemple, obtenir une biomasse et une vigueur suffisante pour assurer une atténuation d'impact des insectes; ou décaler les dates de semis pour favoriser l'évitement, évoqué plus haut.

Ainsi, les grandes lignes de ces itinéraires techniques intégrés sont basées sur des stratégies d'étouffement ou d'évitement et de piège (pour plus de détails voir les actes du CIAG, Mai 2010, ValantinMorison et Pinochet (2010) ou Bouchard et al. (2011)). Les leviers techniques utilisés pour favoriser l'étouffement et l'évitement sont une combinaison des différentes interventions, évoquées dans les premières parties de ce texte, telles que la date de semis, la variété et les mélanges de variétés, l'écartement des lignes de semis, le travail du sol avant semis. De façon à prendre en compte la diversité des conditions pédoclimatiques des sites, en particulier la disponibilité en azote et en eau, deux stratégies sont proposées. La première (stratégie A) propose de favoriser l'évitement et l'atténuation d'impact : ainsi sur sols profonds avec des fortes réserves en eau et surtout en azote un semis précoce ( 15 jours avant la date préconisée dans la région) avec de l'azote disponible permet d'étouffer les adventices, aura aussi pour effet d'éviter la concomitance du phoma (Leptospheria maculans), des limaces ou des insectes d'automne avec des stades sensibles du colza (de cotylédons à 3 feuilles). La deuxième (stratégie $B$ ) repose sur la perturbation du milieu: sur des sols superficiels on favorise la levée puis la destruction des adventices avant la levée du colza par des faux semis répétés. Un mélange variétal ou une bordure de navette sont utilisés pour perturber les insectes dans la recherche de la plante hôte. La combinaison logique de l'itinéraire intégré se construit sur l'ensemble des interventions réalisées pendant le cycle de la culture et se décide lors du choix de la stratégie adoptée. En effet, par exemple lorsque I'on décide de tester un itinéraire technique de type $A$, on a pour objectif de favoriser conjointement l'évitement par la modification de la date de semis et l'atténuation d'impact par une vigueur végétative forte qui permettra au couvert de supporter de fortes attaques de grosses altises, ou de tenthrèdes ou plus tardivement de méligèthes. Décider cela, c'est décider d'aller jusqu'à l'impasse insecticide. Par ailleurs, pour les deux stratégies, le choix variétal s'est avéré fondamental. D'une part, le choix s'est orienté vers une variété au repos végétatif marqué et peu sensible à l'élongation afin d'éviter l'élongation de la tige, que I'on pourrait craindre sur les semis précoce. Ce choix a aussi pour conséquence de ne pas traiter avec un régulateur. Enfin le choix variétal est aussi intervenu pour tenter de piéger des insectes, de printemps et d'appliquer la stratégie de "push-pull " évoquée plus haut. Ce principe de "pusch-pull» a été mis en place en proposant deux solutions : 1) soit une variété tardive à floraison mélangée à une variété précoce à raison de $5 \%$ à $10 \%$ semée en mélange, 2) soit une bordure de navette autour de la parcelle.

Ces itinéraires techniques ont été mis en test en parcelles agricoles entre 2004 et 2011 et ont été évalués sur des critères économiques et environnementaux. Les articles de Valantin-Morison et Pinochet (2010) et Bouchard et al. (2011) et Valantin-Morison et Meynard (2012) montrent que les itinéraires techniques intégrés sur colza permettent un gain environnemental certain (baisse de I'IFT de $40 \%$ ) tout en maintenant une rentabilité pour l'agriculteur: marges brutes et semi-nettes maintenues ainsi qu'une efficience économique supérieure.

Néanmoins, ces travaux sont strictement limités à la parcelle cultivée et la gestion des insectes ravageurs et utiles nécessite de dépasser cette échelle.

\section{Modéliser à l'échelle du paysage}

Afin de répondre encore partiellement à la question de la manière de favoriser la 
régulation biologique à l'échelle du paysage, nous avons entrepris de recourir à la modélisation (Vinatier et Valantin-Morison, 2011). La complexité du système due à l'existence d'interactions non linéaires dans le temps et l'espace entre plusieurs organismes a nécessité une approche de modélisation spatio-temporelle, mécaniste. L'objectif de ce travail de modélisation est de produire un outil pour représenter les effets conjoints de la diversité du paysage agricole, de l'agencement de parcelles agricoles et d'habitats semis naturels et des pratiques appliquées sur le colza sur les dynamiques de populations $d$ 'insectes ravageurs et leur auxiliaires majeurs. Cet outil, d'abord construit pour le couple méligèthesparasitoïdes, a repris tous les éléments de connaissance sur les processus biologiques (démographiques et spatiaux) et les effets des pratiques culturales agissant sur le système, et ceci en interaction avec la configuration paysagère. Le modèle nommé Mosaic-Pest est déterministe et spatialement explicite. L'espace est discrétisé selon une grille de haute résolution. Chaque case de la grille contient un certain effectif des espèces de méligèthes et de parasitoïdes, à un stade (œuf, larve, adulte) et à un état (en vol, en hivernation, en alimentation) donnés. II décrit par des probabilités de transition la variation temporelle des effectifs d'insectes. Les vols sont formalisés par une redistribution spatiale des populations selon un noyau de dispersion dépendant de I'espèce, son stade et de l'attractivité relative des habitats de destination (pour de plus amples détails se référer aux articles en cours, Vinatier et al. (2012a, b)). Ce modèle, encore à l'état de prototype, a été testé et des simulations sur dix ans ont été analysées en modifiant différentes pratiques culturales, comme le travail du sol postrécolte, les bandes pièges, les rotations dans plus de 35 paysages différents. Comparé aux données réelles collectées au champ (Rusch et al., 2011c), le modèle Mosaic-Pest représente de façon satisfaisante la variabilité des données. L'effet positif des prairies sur les taux de parasitismes, phénomène mis en évidence par le modèle, est en accord avec d'autres études indépendantes (Thies et Tscharntke, 1999). L'analyse a révélé un effet dominant du type de paysage à la fois sur les densités du méligèthes et sur le taux de parasitisme et une forte influence des plantes pièges et de la rotation culturale mais un faible effet du labour, contrairement à ce qui était supposé. L'influence de la rotation culturale et du paysage s'explique par une modification de la connectivité des habitats nécessaires à l'accomplissement des cycles des deux espèces étudiées. Les prairies vont jouer le rôle de corridor et augmenter la connectivité des parcelles cultivées. De la même manière, une longue durée de rotation entraînera une augmentation des distances entre précédent colza et parcelle de colza, avec une conséquence négative sur la régulation biologique. Le modèle Mosaic-Pest aide à comprendre les systèmes complexes tels que les relations tri-trophiques à l'échelle paysagère. Chercheurs et agriculteurs peuvent utiliser ce modèle comme un laboratoire virtuel pour tester et sélectionner les meilleures combinaisons de pratiques qui permettront de limiter les populations d'insectes ravageurs, ceci en interaction avec la configuration sachant son importance notable sur les densités de populations. Néanmoins, il est encore en phase de test et d'amélioration et ne pourra pas être utilisé pour identifier des scénarios de protection intégrée à l'échelle d'un paysage avant quelques années.

\section{Conclusion générale}

Afficher l'objectif de favoriser la régulation biologique des bioagresseurs à plusieurs échelles est ambitieux et pourtant incontournable si l'on veut parvenir à concevoir des systèmes de culture économes en pesticides pour cette culture. Vers quelles thématiques de recherche doit-on aller pour espérer atteindre cet objectif ?

On a remarqué tout au long de ce texte que le choix variétale intervenait à de nombreuses reprises autant à l'échelle parcelle que supra parcellaire ; or la sélection variétale ne s'est encore que très peu préoccupée de la recherche de variétés soit " tolérantes ", voire résistantes aux insectes, soit ayant des caractéristiques morphologiques, chimiques, biologiques qui perturbent les relations plantes-insectes. Les résultats encourageants d'études récentes devraient initier de nouvelles thématiques autour de ces questions, car ce levier pourrait être très efficace.

La notion de nuisibilité est apparue également importante car elle pourrait induire de manière très directe une réduction du recours aux solutions chimiques, si des seuils plus objectifs de nuisibilité étaient validés. Des connaissances biologiques suffisamment génériques sont indispensables pour construire des règles de décision qui feraient intervenir l'état du couvert végétal (croissance, vigueur, précocité), I'environnement immédiat de la parcelle, et le nombre d'insectes ravageurs et pourquoi pas certains insectes bénéfiques modèles.

Dépasser la parcelle cultivée, nécessite de recourir à la modélisation spatio-temporelle pour synthétiser les connaissances, parfois pour expliciter les processus majeurs qui expliquent les effets du paysage mesurés et pour tester de différents scénarios de gestion des insectes. La modélisation spatio-temporelle est encore timide mais doit et va sans aucun doute se développer. En outre, analyser, mesurer, quantifier in situ les effets du paysage sur la régulation biologique nécessite également une bonne connaissance des milieux à la fois naturels et anthropisés. Or on doit regretter le manque cruel de connaissances, de banque de données sur les pratiques agricoles des agriculteurs dans un territoire. Seules des enquêtes lourdes, spatialisées permettent de connaître ne serait ce que les espèces, variétés, travaux du sol réalisés.

De manière générale, il est frappant de constater le manque criant de connaissances biologiques, écologiques sur beaucoup d'insectes ravageurs des cultures (dont le colza) et pire sur les insectes auxiliaires (y compris une majorité de carabes). Ces connaissances seraient utiles à bien des niveaux, pour interpréter les résultats expérimentaux, pour éclairer les sélectionneurs variétaux, pour améliorer les seuils de nuisibilité, pour modéliser les influences du paysage. Néanmoins, cela ne résoudra pas tout. En effet, afficher un tel objectif nécessite d'une part une vision systémique de l'agro-système à une échelle bien plus grande que celle de la parcelle cultivée et d'autre part requiert une démarche et des méthodes d'ingénierie. L'ensemble de ces travaux constituent donc un front de recherche majeur et permettra sûrement de conjuguer agronomie et écologie et de construire les bases d'une discipline naissante qu'est l'agro-écologie. L'écologie, par son autre regard sur le milieu, par la multitude de 
ces concepts et de ces méthodes d'analyse enrichira sans aucun doute l'analyse de la composante biologique en milieu anthropisé. Mais n'oublions pas que l'agronomie adopte une vision systémique depuis toujours et se concentre de manière grandissante sur la composante biologique du champ cultivé ; I'agronomie a des approches territoriale et lie agronomie et environnement depuis longtemps également quand elle évalue la durabilité des systèmes de cultures à l'échelle du territoire (Meynard et al., 2003). Enfin, I'agronomie est aussi une science pour l'action, ce qui pourrait alors permettre de traduire, de transformer les connaissances fondamentales, évoquées souvent ici, en clefs d'action pour des systèmes de culture économes en pesticides.

Remerciements. Un certain nombre d'expérimentations ont été réalisées dans notre laboratoire et sont illustrées ici ; nous remercions donc l'ensemble des techniciens qui ont assuré le suivi de ces expérimentations et tout particulièrement, Gilles Grandeau, Arnaud Butier. En outre, un certain nombre d'agriculteurs ont accepté d'accueillir des essais, d'expliquer leurs pratiques, parfois de prendre des risques, nous leurs en sommes également reconnaissants. Enfin, de chaleureux remerciements et encouragements à tous les conseillers de chambres d'agriculture qui accompagnent ces agriculteurs innovants et qui s'engagent avec eux sur le chemin de la protection intégrée.

\section{RÉFÉRENCES}

Alford DV, Nilsson C, Ulber B. Insect pests of oilseed rape crops. In : DV Alford (Ed.), Biocontrol of oilseed rape pests. Oxford, UK : Blackwell Science, 2003: 9-41.

Altieri MA. The ecological role of biodiversity in agroecosystems. AgriC Ecosyst Environ 1999; $74: 19-31$.

Altieri MA, Nicholls $\mathrm{Cl}$ An agroecological basis for biological control through conservation. In : California conf on biological control IV. Berkeley, California, USA, 13-15 July 2004 : 2839.

Andersen MK, Hauggaard-Nielsen H, Weiner J, Jensen ES. Competitive dynamics in twoand three-component intercrops. I Appl Ecol 2007 ; 44 : 545-51.

Andow DA. Vegetational diversity and arthropod population response. Annu Rev Entomol $1991 ; 36: 561-86$.
Attoumani-Ronceux A, Aubertot JN, Guichard L, et al. Guide pratique pour la conception de systèmes de culture économes en produits phytosanitaires - Application aux systèmes de polyculture. Edition MAAPRAT, 2011. Document principal et documents annexes disponibles en téléchargement sur: www.systemesdecultureinnovants.org/et sur http://agriculture.gouv.fr/guide-ecophytograndes-cultures

Aubertot JN, Carpentier A, Gril JJ, et al. Pesticides, agriculture et environnement: réduire I'utilisation des pesticides et limiter leurs impacts environnementaux. Expertise collective INRA-Cemagref, 2005.

Barari H, Cook SM, Clark SJ, Williams IH. Effect of a turnip rape (Brassica rapa) trap crop on stem-mining pests and their parasitoids in winter oilseed rape (Brassica napus). BioControl 2005 ; 50 : 69-86.

Barbottin A, Le Bail M, Jeuffroy MH. The Azodyn crop model as a decision support tool for choosing cultivars. Agronomy for Sustainable Development 2006 ; 26 : 107-15.

Bianchi FJJA, Booij CJH, Tscharntke T. Sustainable pest regulation in agricultural land- scapes: A review on landscape composition, biodiversity and natural pest control. PRoy Soc Lond 2006 ; 273 : 1715-27.

Bjorkman C, Eklund K. Factors affecting willow leaf beetles (Phratora vulgatissima) when selecting overwintering sites. Agricultural and Forest Entomology 2006 ; 8 : 97-101.

Bouchard C, Valantin-Morison M, Grandeau G. Itinéraires techniques intégrés du colza d'hiver : comment concilier environnement et économie. Courrier de l'environnement. Inra, décembre 2011.

Broad ST, Schellhorn NA, Lisson SN, Mendham NJ, Corkrey R. Host location and parasitism of Brevicoryne brassicae in diversified broccoli cropping systems. Entomol Exp Appl 2008 ; 129 : 166-71.

Büchi R. Mortality of pollen beetle (Meligethes spp.) larvae due to predators and parasitoids in rape fields and the effect of conservation strips. Agriculture Ecosystems and Environment 2002 ; 90 : 255-63.

Büchs W. Impact of on-farm landscape structures and farming systems on predators. In: DV Alford (Ed.) Biocontrol of oilseed rape pests. Oxford, UK : Blackwell, 2003.

Büchs W, Harenberg A, Prescher S, Weber G, Hattwig F. Entwicklung von Evertebratenzonosen bei verschiedenen Formen der Flachenstillegung und Extensivierung. Mitteilungen aus der Biologischen Bundesanstalt fur Landund Forstwirtschaft Berlin-Dahlem 1999 ; $368: 9-38$.

Büchs W, Katzur K. Cultivation techniques as means to control pests in organic oilseed rape production. IOBC/wprs Bull $2004 ; 27: 225$ 36.

Butts RA, Floate KD, David M, Blackshaw RE, Burnett PA. Influence of intercropping canola or pea with barley on assemblages of ground beetles (Coleoptera: Carabidae). Environ Ento$\mathrm{mol} 2003 ; 32$ : 535-41.

CETIOM, 2011. http://www.cetiom.fr/dossiers-phares/techniques-innovantes-dimplantations/couverts-associes

Cook SM, Bartlet E, Murray DA, Williams IH. The role of pollen odour in the attraction of pollen beetles to oilseed rape flowers. Entomol Exp Appl 2002 ; 104 : 43-50.

Cook SM, Khan ZR, Pickett JA. The use of push-pull strategies in integrated pest management. Ann Rev Entom 2007 ; 52 : 375-400.

Cook SM, Smart LE, Martin JL, Murray DA, Watts NP, Williams IH. Exploitation of host plant preferences in pest management strategies for oilseed rape (Brassica napus). Entomologia Experimentalis et Applicata 2006 ; 119 : 221-9.

David C, Jeuffroy MH, Laurent F, Mangin M, Meynard JM. The assessment of Azodyn-Org model for managing nitrogen fertilization of organic winter wheat. Eur J Agron $2005 ; 23$ : 225-42.

Denys C, Tscharntke T. Plant-insect communities and predator-prey ratios in field margin strips, adjacent crop fields, and fallows. Oecologia $2002 ; 130$ : 315-24

Dosdall LM, Herbut MJ, Cowle NT. Susceptibilities of species and cultivars of canola and mustard to infestation by root maggots (Delia spp.) (Diptera: Anthomyiidae). Canadian Entomologist 1994 ; 126 : 251-60.

Dosdall LM, Herbut MJ, Cowle NT, Micklich TM. The effect of seeding date and plant density on infestations of root maggots, Delia spp. (Diptera: Anthomyiidae), in canola. Canadian Journal of Plant Science 1996 ; $76: 169-77$.

Dosdall LM, Stevenson FC. Managing flea beetles (Phyllotreta spp.) (Coleoptera: Chrysomelidae) in canola with seeding date, plant density, and seed treatment. Agronomy Journal 2005 ; 97 : 1570-8.

Dosdall LM, Dolinski MG, Cowle NT, Conway PM. The effect of tillage regime, row spacing, and seeding rate on feeding damage by flea beetles, Phyllotreta spp. (Coleoptera: Chrysomelidae), in canola in central Alberta, Canada. Crop Protection 1999 ; 18 : 217-24.

Dunning JB, Danielson JB, Pulliam HR. Ecological processes that affect populations in complex landscapes. Oikos $1992 ; 65$ : 169-75.

Ecophyto R\&D. Vers des systèmes de culture économes en produits phytosanitaires. Tome II. Analyse comparative des différents systè- 
mes en grandes cultures. Paris : Inra éditeur (France), 2009.

Eickermann M, Ulber B, Vidal S. Resynthesized lines and cultivars of Brassica napus $\mathrm{L}$. provide sources of resistance to the cabbage stem weevil (Ceutorhynchus pallidactylus (Mrsh.)). Bulletin of Entomological Research 2011; 101: 287-94.

Eilenberg J, Hajek A, Lomer C. Suggestions for unifying the terminology in biological control. Biocontrol $2001 ; 46: 387-400$.

Ellis PR, Farrell JA. Resistance to cabbage aphid (Brevicoryne brassicae) in six brassica accessions in New Zealand. New Zealand Journal of Crop and Horticultural Science 1995 ; 23 : 25-9.

Fahrig L, Merriam G. Conservation of fragmented populations. Conserv Biol 1994 ; 8 : 50-9.

Ferron P, Deguine JP. Crop protection, biological control, habitat management and integrated farming, a review. Agron Sustain Dev $2005 ; 25$ : 17-24.

Finch S, Collier RH. Host-plant selection by insects - a theory based on 'appropri- ate/ inappropriate landings' by pest insects of cruciferous plants. Entomol Exp Appl 2000 ; 96: 91-102.

Finch S, Collier RH. Host-plant selection by insects - the 'missing link'. IOBC/wprs Bull $2003 ; 26: 103-8$.

Frank T. Species diversity and activity densities of epigaeic and flower visiting arthropods in sown weed strips and adjacent fields. Bulletin OILB/SROP 1996 ; 19 : 101-5.

Free JB, Williams IH The response of pollen beetle, Meligethes aeneus, and the seed weevil, Ceutorhynchus assimilis, to oilseed rape, Brassica napus, and other plants. / App/ Ecol 1978; 15: 761-74.

Gurr GM, Wratten SD, Luna JM. Multifunction agricultural biodiversity: pest management and other benefits. Basic Appl Ecol $2003 ; 4$ : 107-16.

Hokkanen HMT. Biological control methods of pest insects in oilseed rape. EPPO Bull 2008 ; 38 : 104-9.

Hokkanen HMT, Husberg GB, Soderblom M. Natural enemy conservation for the integrated control of the rape blossom beetle Meligethes aeneus F. Ann Agr Fenn 1988 ; 27 : 281-94.

Holland JM. The environmental consequences of adopting conservation tillage in Europe: reviewing the evidence. Agric Ecosyst Environ $2004 ; 103: 1-25$.

Hopkins RJ, Ekbom B. Low oviposition stimuli reduce egg production in the pollen beetle Meligethes aeneus. Physiol Entomol 1996 ; 21 : 118-22.

Hopkins RJ, Ekbom B. The pollen beetle, Meligethes aeneus, changes egg production rate to match host quality. Oecologia 1999 ; $120: 274-8$.

Horrigan L, Lawrence RS, Walker P. How sustainable agriculture can address the environmental and human health harms of industrial agriculture. Environmental Health Perspectives 2002 ; 110 : 445-56.

Hummel JD, Dosdall LM, Clayton GW, Harker KN, O'Donovan JT. Responses of the parasitoids of Delia radicum (Diptera: Anthomyiidae) to the vegetational diversity of intercrops. Biological Control $2010 ; 55$ : 151-8.

Jullien A, Mathieu A, Allirand JM, et al. Characterization of the interactions between architecture and source-sink relationships in winter oilseed rape (Brassica napus) using the GreenLab model. Ann Bot 2011; 107 : 765-79.

Kogan M. Plant resistance in pest management. In: RL Metcalf, WH Luckmann (Eds.), Introduction to Insect Pest management, 3rd Edition. New York: Wiley, 1994 : 73128.

Kromp B. Carabid beetles in sustainable agriculture: a review on pest control efficacy, cultivation impacts and enhancement. Agriculture Ecosystems \& Environment $1999 ; 74$ : 187-228.

Lagerlof J, Wallin H. The abundance of arthropods along two field margins with different types of vegetation composition: an experimental study. Agriculture Ecosystems \& Environment 1993; 43 : 141-54.

Lançon J, Wery J, Rapidel B, et al. An improved methodology for integrated crop management systems. Agronomy for Sustainable Development 2007 ; 27 : 101-10.

Landis DA, Wratten SD, Gurr GM. Habitat management to conserve natural enemies of arthropod pests in agriculture. Annu Rev Entomol 2000 ; 45 : 175-201.

Lerin J. Yield losses associated with 2 successive pests (Ceuthorrhynchus napi Gyll. and Meligethes aeneus $F$ ) on winter rape (cultivar Bienvenu). Agronomie 1988 ; 8 : 251-56.

Loyce C, Rellier JP, Meynard JM. Management planning for winter wheat with multiple objectives: The BETHA system. Agricultural Systems $2002 ; 72: 9-31$.

Mabbett T. Straw incorporation trials reveal arable slug damage will increase. Agr Int 1991 ; 43 : 304-6.

Malézieux E, Crozat Y, Dupraz C, et al. Mixing plant species in cropping systems: concepts, tools and models. A review. Agronomy for Sustainable Development 2008; 28. e-first : DOI: 10.1051/agro2007057.

Marshall EJP. Agricultural landscapes: Field margin habitats and their interaction with crop production. J Crop Improvement 2004 ; 12 : 365-404.

Matson PA, Parton WJ, Power AG, Swift MJ. Agricultural intensification and ecosystem properties. Science 1997 ; 277 : 504-9.

Médiène $S$, Valantin-Morison M, Sarthou JP, et al. Agroecosystem management and biotic interactions: a review ". Agronomy for Sustainable Development 2011 ; 31 : 491514.

Meynard JM, Doré T, Habib R. L'évaluation et la conception de systèmes de culture pour une agriculture durable. Les Entretiens du Pradel 1 ère édition. Autour d'Olivier de Serres: Pratiques Agricoles et Pensée Economique Partie Agronomique ; 2000/09/28-30 ; Le Pradel (FRA). Comptes Rendus de l'Académie d'Agriculture de France 2001 ; 87 : 223-36.

Meynard JM, Doré T, Lucas P. Agronomic approach: cropping systems and plant diseases. Comptes Rendus Biologies 2003;37-46.

Nilsson C. Impact of ploughing on emergence of pollen beetle parasitoids after hibernation. Z Angew Entomol $1985 ; 100$ : 302-8.

Nilsson C. Pollen Beetle (Meligethes aeneus spp) in oilseed rape crops (Brassica napus L.): Biological interactions and crop losses. Dissertation. Sweden: Swedish University of Agricultural Sciences, 1994.

Pfiffner L, Wyss E. Use of sown wildflower strips to enhance natural enemies of agricultural pest. In : Gurr GM, et al., (Eds.), Ecological engineering for pest management Advances in habitat manipulation for arthropods. CSIRO Publishing - CABI Publishing, Collingwood - Oxon, 2004 : 165-86.

Pickett $\mathrm{CH}$, Bugg RL. Enhancing biological control: Habitat management to promote natural enemies of agricultural pests. Berkeley, USA : University of California Press, 1998.

Pickett CH, Roltsch W], Corbett A, Daane KM. Habitat management for enhancing biological control: benefits and pitfalls. In: California Conference on Biological Control II, The Historic Mission Inn Riverside, California, USA, 11-12 July 2000: 81-5.

Pilorgé E, Maisonneuve C, Ballanger Y. Les ravageurs du colza d'hiver. Paris : CETIOM, 1997: 75-85.

Plantegenest M, Le May C, Fabre F. Landscape epidemiology of plant diseases. Journal of the Royal Society 2007 ; 4 : 963-72.

Podlaska J, Markus J, Dmoch J, Loboda T. Compensation of the damage caused by pollen beetle (Meligethes aeneus) on winter oilseed rape under different plant density and fertilisation. I - some morphological characters. Rosliny Oleiste 1996 ; 17 : 325-30.

Pontin DR, Wade MR, Kehrli P, Wratten SD. Attractiveness of single and multiple species 
flower patches to beneficial insects in agroecosystems. Ann App Biol 2006 ; 148 : 39-47.

Potting RPJ, Perry JN, Powell W. Insect behavioural ecology and other factors affecting the control efficacy of agro-ecosystem diversification strategies. Ecological Modelling $2005 ; 182$ : 199-216.

Prévost $P$. La régulation biologique un concept intégrateur de la connaissance agronomique. Courrier de l'environnement de I'INRA 2000; (39): 27-35.

Pullaro TC, Marino PC, Jackson DM, Harrison HF, Keinath AP. Effects of killed cover crop mulch on weeds, weed seeds, and herbivores. Agric Ecosyst Environ 2006 ; 115 : 97-104. DOI :10.1016/j.agee.2005.12.021.

Rahim A, Hashmi A, Khan NA. Effects of temperature and relative humidity on longevity and development of Ooencyrtus papilionis Ashmead (Hymenoptera: Eulophidae), a parasite of the sugarcane pest, Pyrilla perpusilla Walker (Homoptera: Cicadellidae). Environmental Entomology 1991 ; 20 : 774-5.

Rebek EJ, Sadof CS, Hanks LM. Influence of floral resource plants on control of an armored scale pest by the parasitoid Encarsia citrina (Craw.) (Hymenoptera: Aphelinidae). Biological Control 2006 ; 37 : 320-8.

Riechert SE, Lockley T. Spiders as biological control agents. Annu Rev Entomol $1984 ; 29$ : 299-320.

Roland J, Taylor PD. Insect parasitoid species respond to forest structure at different spatial scales. Nature 1997 ; 386 : 710-13.

Root RB. Organisation of a plant-arthropod association in simple and diverse habitats : the fauna of collards (Brassica oleracea). Ecol Monogr $1973 ; 43$ : 95-124.

Rusch A, Valantin-Morison M, Sarthou JP, Roger-Estrade J. Biological control of insect pests in agroecosystems: effects of crop management, farming systems and seminatural habitats at the landscape scale. A review. Advances in Agronomy 2010 : 219-259.

Rusch A, Valantin-Morison M, Sarthou JP, Roger-Estrade J. Multi-scale effects of landscape complexity and crop management on pollen beetle parasitism rate. Landscape Ecology 2011a ; 26 : 473-86.

Rusch A, Valantin-Morison M, Sarthou JP, Roger-Estrade J. Effect of crop management and landscape context on insect pest populations and crop damage. Agriculture, Ecosystems and Environment 2011b (in press; http://dx.doi.org/10.1016/j.agee. 2011. 05.004)

Rusch A, Valantin-Morison M, Sarthou JP, Roger-Estrade J. Local and landscape determinants of pollen beetle (Meligethes aeneus F) abundance in overwintering habitats. Agriculture and Forest Ecology 2011c.
Rusch A, Valantin-Morison M. Effect of nitrogen fertilization, cultivar and species on attractiveness and nuisibility of two major pests of winter oilseed rape (Brassica napus L.): pollen beetle (Meligethes aeneus F) and stem weevil (Ceutorhynchus napi Gyl.). Congress of European Society Agronomy. 15-18 Septembre 2008. Bologne (Italie).

Schmidt MH, Thewes U, Thies C, Tscharntke $\mathrm{T}$. Aphid suppression by natural enemies in mulched cereals. Entomologia Experimentalis et Applicata $2004 ; 113$ : 87-93.

Sotherton NW. The distribution and abundance of predatory arthropods overwintering on farmland. Annals of Applied Biology 1984 ; $105: 423-9$.

Stern VM, Smith RF, Van den Bosch F, Hagen $\mathrm{KS}$. The integrated control concept. Hilgardia $1959 ; 29: 81-101$.

Stoate C, Boatman ND, Borralho RJ, Carvalho CR, de Snoo GR, Eden P. Ecological impacts of arable intensification in Europe. J Environ Manage 2001 ; 63 : 337-65.

Tahvanainen JO, Root RB. The influence of vegetational diversity on the population ecology of a specialized herbivore, Phyllotreta cruciferae (Coleoptera: Chrysomelidae). Oecologia 1972; 10 : 321-46. DOI : 10.1007/BF00345736.

Thies C, Steffan-Dewenter I, Tscharntke T. Effects of landscape context on herbivory and parasitism at different spatial scales. Oikos $2003 ; 101: 18-25$.

Thies C, Tscharntke T. Landscape structure and biological control in agroecosystems. Science 1999 ; 285 : 893-95.

Thorbek P, Bilde T. Reduced numbers of generalist arthropod predators after crop management / Appl Ecol 41:526-38.

Tilman D, Cassman KG, Matson PA, Naylor R, Polasky S. Agricultural sustainability and intensive production practices. Nature 2002 ; $418: 671-7$

Ulmer B], Dosdall LM. Emergence of overwintered and new generation adults of the crucifer flea beetle, Phyllotreta cruciferae (Goeze) (Coleoptera: Chrysomelidae). Crop Protection 2006 ; 25 : 23-30.

Valantin-Morison M, Meynard JM. A conceptual model to design prototypes of crop management: a way to improve organic Winter Oilseed Rape performance in a survey in farmers' fields. In: Crop Management, ed In TECH, 2012.

Valantin-Morison M, Quere L. Effects of turnip rape trap crops on oilseed rape pests in a network of organic farmers' fields. Congress of European Project MASTER, Integrated pest mangement Göttingen (Allemagne), 2006/04/2-5
Valantin-Morison M, Guichard L, Jeuffroy $\mathrm{MH}$. Comment maîtriser la flore adventice des grandes cultures à travers les éléments de l'itinéraire technique ? Carrefour de l'Innovation Agronomique 2008 ; 3 : 27-41. http:// www.inra.fr/ciag/revue/volume_3_decembre_ 2008

Valantin-Morison M, Butier A, Berder J. Mixing Winter oilseed rape (WOSR) and legume to smoother weeds, disturb insects and reduce nitrogen use in spring: possible or not? 13th International Rapeseed Congress June 5-9, 2011. Prague, Czech Republic.

Valantin-Morison $\mathrm{M}$, Pinochet $\mathrm{X}$. Concevoir des itinéraires techniques intégrés pour le colza d'hiver: les acquis et les défis de demain. Carrefour de I'Innovation Agronomique $2010 ; 8$ : 35-55. http://www.inra.fr/ ciag/revue/volume_8_mai_2010

Valantin-Morison M, Meynard J, Dore T. Effects of crop management and surrounding field environment on insect incidence in organic winter oilseed rape (Brassica napus L.). Crop Protection 2007 ; 26 : 110820. doi :10.1016/j.cropro.2006.10.005.

Vinatier F, Valantin-Morison M. Mosaic-Pest: Landscape management of the pollen beetle using a spatially explicit model. In: Proceedings of the 8th World Congress of the International Association for Landscape Ecology. 18-23 august. Beijing, China, 2011 : 545-46.

Vinatier $F$, Gosme $M$, Valantin-Morison $M$ Mosaic-Pest, landscape model for pest management(I): model description and use for IPM strategies testing. Lanscape Ecology 2012a (soumis)

Vinatier F, Gosme M, Valantin-Morison M. Mosaic-Pest, landscape model for pest management(II): parameter estimation and sensitivity analysis. Landscape ecology 2012b (soumis).

Vinatier F, Valantin-Morison M. How to reinforce pollen beetle biocontrol at landscape level using a spatially explicit model ? IOBC 3rd-5th october 2011. Göttingen

Vinatier $F$, Tixier $P$, Duyck $P H$, Lescourret $F$. Factors and mechanisms explaining spatial heterogeneity: a review of methods for insect populations. Methods in Ecology and Evolution $2011 ; 2: 11-22$

Wackers FL. Assessing the suitability of flowering herbs as parasitoid food sources: flower attractiveness and nectar accessibility. Biological Control 2004 ; 29 : 307-14.

Weiss MI, Schatz BG, Gardner IC, Nead BA. Flea beetle (Coleoptera: Chrysomelidae) populations and crop yield in field pea and oilseed rape intercrops. Environ Entomol 1994 ; $23: 654-58$

Williams IH Free JB. Compensation of oil seed rape (Brassica napus $L$.) plants after damage 
to their buds and pods. J Agr Sci 1979; 92: 53-9.

Williams IH (ed.). Biocontrol-Based Integrated Management of Oilseed Rape Pests. Dordrecht: Springer Netherlands, 2010a.

Williams IH. The major insect pests of oilseed rape in Europe and their management: An overview. In: Williams IH (Eds.), Biocontrol-based integrated management of oilseed rape pests. Springer London, 2010b : 1-44.
Wyss E. The effects of weed strips on aphids and aphidophagous predators in an apple 14871488 orchard. Entomol Exp Appl 1995; 75: 43-9.

Zabel J, Tscharntke T. Does fragmentation of Urtica habitats affect phytophagous and predatory insects differentially? Oecologia $1998 ; 116: 419-25$.

Zaller JG, Moser D, Drapela T, Schmoger C, Frank T. Effect of within-field and landscape factors on insect damage in winter oilseed rape. Agr Ecosyst Environ 2008 ; 123 : 23338.

Zaller JG, Moser D, Drapela T, Schmoger C, Frank $\mathrm{T}$. Insect pests in winter oilseed rape affected by field and landscape characteristics. Basic Appl Ecol 2008 ; 9 : 682-90.

Zehnder GW, Hough-Goldstein J. Colorado potato beetle (Coleoptera: Chrysomelidae) population development and effects on yield of potatoes with and without straw mulch. J Econ Entomol 1989 ; 83 : 1982-7. 